\title{
Ocorrência de Anisakis simplex (Nematoda: Anisakidae) em bacalhau comercializado em Volta Redonda, Rio de Janeiro, Brasil
}

\author{
Anisakis simplex (nematoda: anisakidae) occurrence in codfish \\ commercialized in Volta Redonda, Rio de Janeiro, Brazil
}

1 Daniela da Silva Santos

2 Dimitri Ramos Alves dimitri_ralves@yahoo.com.br

1 Graduada em Nutrição, UniFOA.

2 Docente do Centro Universitário de Volta Redonda, UniFOA.

\section{RESUMO}

Nas últimas décadas, houve um aumento significativo dos casos de zoonoses transmitidas pelo consumo de pescado cru ou mal cozido, no Brasil. Entre julho e agosto de 2015, foram adquiridos 10 espécimes de bacalhau (Gadus morhua) (Osteichthyes: Gadiformes: Gadidae) provenientes de supermercados do Município de Volta Redonda, Estado do Rio de Janeiro, Brasil. Os espécimes foram analisados com o objetivo de identificar e registrar os parasitos de potencial zoonótico. As análises do pescado foram realizadas no Laboratório de Técnica Dietética do Curso de Nutrição UniFOA. Foi constatada a presença de larvas de Anisakis simplex $\left(\mathrm{L}_{3}\right)$ em todas as amostras examinadas. Um total de 652 larvas foi coletado, com média de 65,2 $\pm 33,7(17-113)$ parasitos por amostra examinada. As larvas encontravam-se encapsuladas, enroladas em espiral plana, sob o peritônio e a pele e inseridos na musculatura das amostras de bacalhau. A presença dessas larvas nas amostras do bacalhau analisadas as torna impróprias para o consumo.

\section{Palavras-chave}

Bacalhau; Gadus morhua; Anisakidae; Anisakis simplex; Anisaquíase.

\begin{abstract}
In the last decades there was been a significant increase in the number of cases of Borne zoonoses by consumption of raw or undercooked fish in Brazil. From July and August 2015, 10 specimens of codfish (Gadus morhua), (Osteichthyes: Gadiformes: Gadidae) were acquired from supermarkets in the town of Volta Redonda city, State of Rio de Janeiro, Brazil. The codfish samples were analyzed with purpose to identify and record the potential zoonotic parasites. The codfish analyzes were performed in The laboratory of the Nutrition Course Centro Universitário de Volta Redonda. The presence of Anisakis simplex larvae (L3) in all samples examined was observed. Six hundred fifty-two larvae, with an averange of 65.2 $\pm 33.7(17-113)$ per samples, were collected. The larvae were encapsulated, flat spiral wound, under the peritoneum and skin and inserted in the musculature of codfish samples. The presence of these larvae in codfish samples analyzed makes them unfit for consumption.
\end{abstract}

\section{Keywords}

Codfish; Gadus morhua; Anisakidae; Anisakis simplex;

\section{Como você deve citar?}

SANTOS, Daniela da Silva; ALVES, Dimitri Ramos. Ocorrência de Anisakis simplex (nematoda: anisakidae) em bacaIhau comercializado em Volta Redonda, Rio de Janeiro, Brasil Cadernos UniFOA, Volta Redonda, n. 31, p. 131-140, ago. 2016. 
Anisakiasis.

\section{INTRODUÇÃO}

As Doenças Transmitidas por Alimentos (DTA) constituem um dos problemas de saúde pública mais frequente do mundo contemporâneo. No Brasil, somente alguns estados e/ou municípios dispõem de estatísticas e dados sobre a ocorrência de surtos de DTA, agentes etiológicos mais comuns, alimentos mais frequentemente implicados, população de maior risco e fatores contribuintes (SANTOS, 2010). Nas últimas duas décadas, observamos uma modificação no perfil das DTAs brasileiras consideradas zoonoses, as quais eram representadas principalmente pela Toxoplasmose e pelo complexo TeníaseCisticercose, com a introdução das parasitoses transmitidas pela ingestão do pescado (ROSSI et al., 2014). Essa modificação no perfil pode ser constatada ao analisarmos a lista revisada do Ministério da Saúde do Brasil, que inclui preponderantemente agentes biológicos com risco para o homem e para a saúde pública, entre os quais se encontram alguns com potencial zoonótico transmitidos pela ingestão do pescado (BRASIL, 2010). Tais agentes biológicos foram registrados ocorrendo em uma ampla variedade de espécies de peixes marinhos e dulcícolas, bivalves e cefalópodes consumidos no território brasileiro (OKUMURA et al., 1999; LUQUE, POULIN, 2004; CARDOSO et al., 2006; TAVARES, LUQUE, 2006; SAAD, LUQUE, 2009; SAAD et al., 2012; MATTOS et al., 2014).

No Brasil, os registros de casos das parasitoses transmitidas via ingestão do pescado aumentaram significativamente. Dentre esses, podemos destacar a ocorrência da Anisaquíase (NETO et al., 2007; CRUZ et al., 2010) Clonorquíase (LEITE et al., 1989) em imigrantes asiáticos, Difilobotríase (EDUARDO et al.; 2005a; 2005b; SANTOS, FARO, 2005; TAVARES et al., 2005; EMMEL et al., 2006; CAPUANO et al., 2007) e da Fagicolose (CHIEFFI et al., 1990, 1992).

Estudos sobre a ocorrência de parasitos em espécimes de bacalhau são escassos no Brasil. Os estudos existentes foram realizados por Pereira et al. (2000) e por Prado e Capuano (2006), ambos no Estado de São Paulo, e, recentemente, por Mafra et al. (2015) com bacalhau proveniente do Estado de Minas Gerais.

O presente estudo tem como objetivo identificar e registrar os parasitos de potencial zoonótico localizados na musculatura somática dos espécimes de bacalhau (eviscerados, salgados e secos) comercializados no Município de Volta Redonda, Rio de Janeiro, Brasil.

\section{MATERIAL E MÉTODOS}

Entre julho e agosto de 2015, foram adquiridos 10 espécimes de bacalhau (Gadus morhua) (Osteichthyes: Gadiformes: Gadidae), totalizando $18,750 \mathrm{Kg}$ de pescado, provenientes de supermercados do Município de Volta Redonda, Estado do Rio de Janeiro, Brasil. Os espécimes estavam eviscerados, secos e salgados (Fig. 1). As análises e inspeção do pescado foram realizadas no Laboratório de Técnica Dietética do Curso de Nutrição do Centro Universitário de Volta Redonda (UniFOA). As amostras foram demolhadas por um período de 24 horas para realização da análise parasitológica. Para a coleta dos parasitos, a musculatura somática dos peixes foi filetada, desfiada e inspecionada, com auxílio de um estereomicroscópio por transparência/transiluminação. Os espécimes de nematoides foram coletados, fixados em AFA (Álcool-Formalina-Ácido acético) e, 48 horas depois, transferidos para preservação em etanol $70^{\circ} \mathrm{GL}$. A clarificação dos espécimes foi feita com Lactofenol de Amann. A coleta e processamento dos nematoides foram feitos de acordo com os procedimentos indicados por Amato et al. (1991) e Eiras et al. (2000). As larvas foram identificadas segundo Petter e Maillard (1988) e Tavares e 
Luque (2006). O processamento e a identificação dos nematoides foram realizados no Laboratório de Parasitologia do UniFOA.

Figura 1 - Espécime de bacalhau (Gadus morhua) eviscerado, seco e salgado. Foto: Autores.

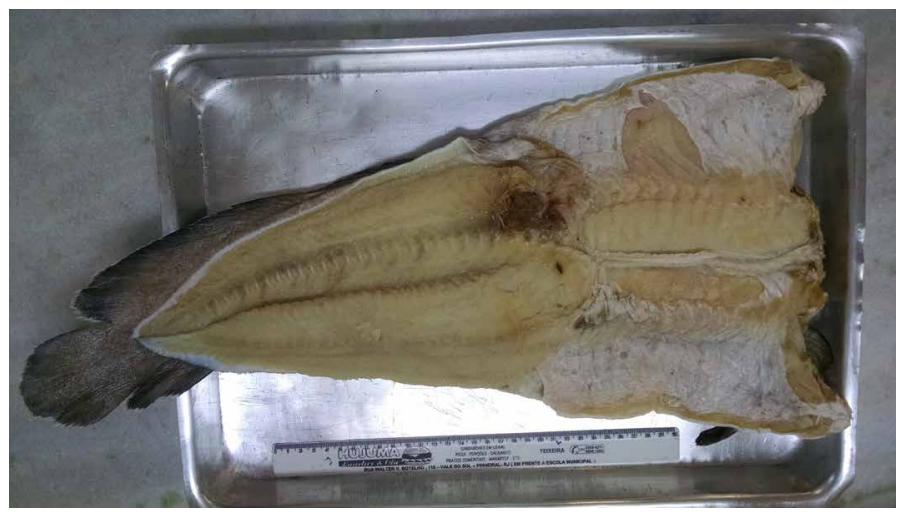

Fonte: dos autores.

\section{RESULTADOS E DISCUSSÃO}

Foi constatada a presença de larvas de Anisakis simplex $\left(\mathrm{L}_{3}\right)$ em todas as dez amostras examinadas, que estavam mortas. Os espécimes de nematoides foram detectados por inspeção visual efetuada durante a dissecção e o pelo processo de transiluminação das amostras (Fig. 2). Um total de 652 larvas foi coletado, com média de 65,2 $\pm 33,7$ (17 - 113) parasitos por amostra examinada. As larvas encontravam-se encapsuladas, enroladas em espiral plana, sob o peritônio e a pele e inseridos na musculatura (Figs. 3, 4, 5, e 6), distribuídas por todo o pescado.

Figura 2 - Espécime de bacalhau demolhado com a presença das larvas de Anisakis simplex observadas por transiluminação.

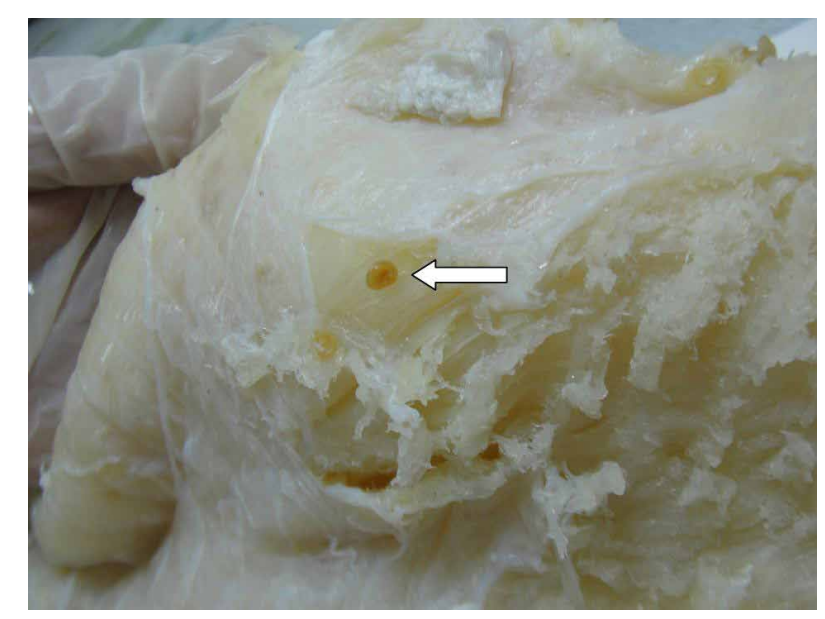

Fonte: dos autores. 
Figura 3 - Larvas de Anisakis simplex enroladas em espiral plana na musculatura do espécime de bacalhau.

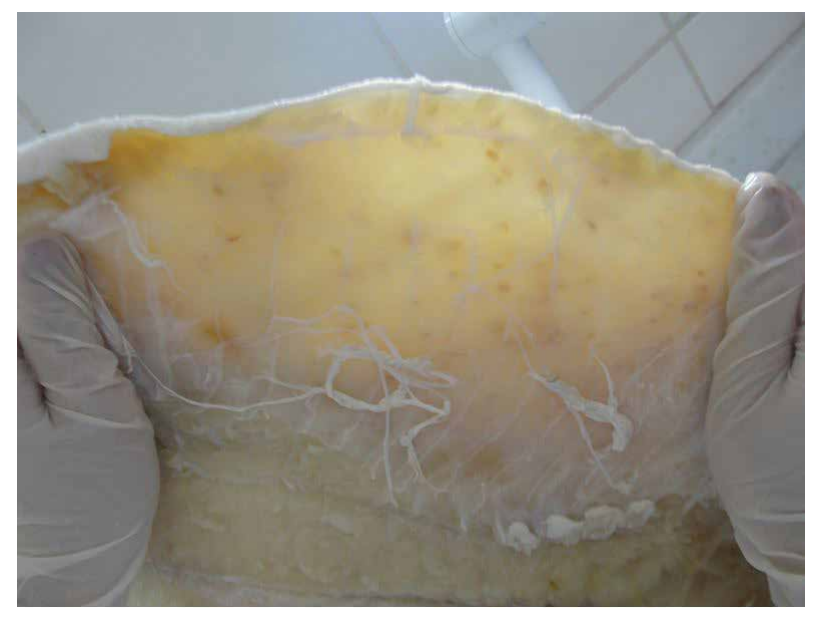

Fonte: dos autores.

Figura 4 - Larvas de Anisakis simplex enroladas em espiral plana sob o peritôneo do espécime de bacalhau.

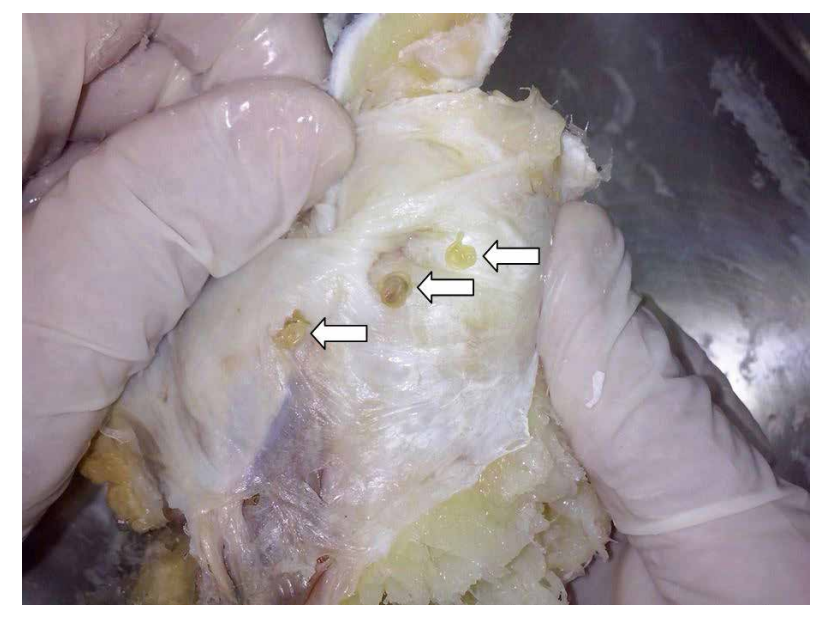

Fonte: dos autores.

Figura 5 - Larvas de Anisakis simplex na musculatura do espécime de bacalhau.

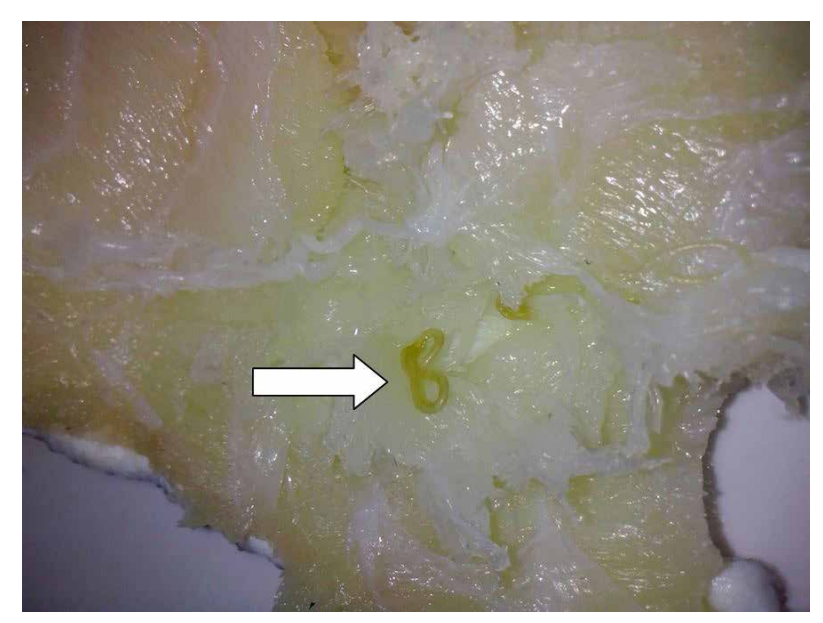

Fonte: dos autores. 
Figura 6 - Espécimes de Anisakis simplex (larvas) coletados na musculatura do bacalhau.

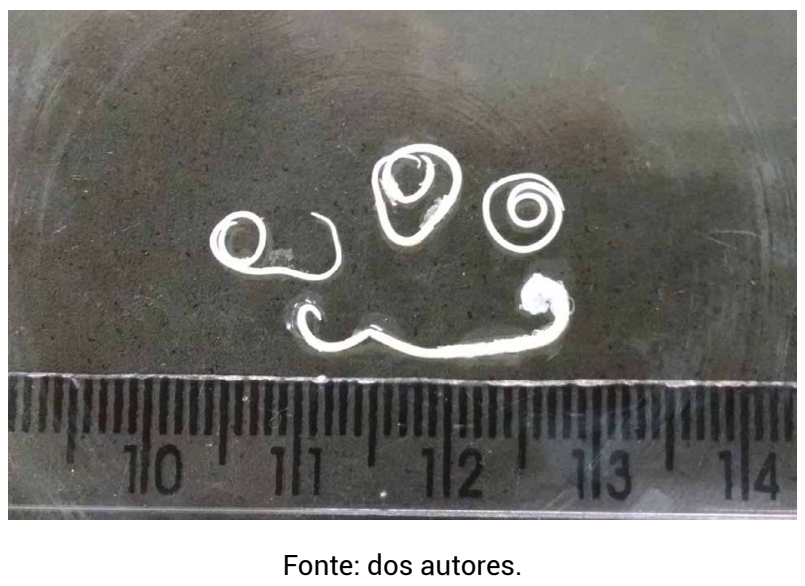

Os resultados obtidos no presente estudo estão em concordância com a literatura sobre a ocorrência de larvas de anisaquídeos (Nematoda: Anisakidae) em bacalhau. Os estudos realizados no Brasil (PEREIRA et al., 2000; PRADO, CAPUANO, 2006) e em Portugal (RAMOS, 2011) indicam que essas larvas são comumente registradas em espécimes de bacalhau (Gadus morhua e Gadus macrocephalus). Nos estudos de Pereira et al. (2000), Prado e Capuano (2006) e Ramos (2011), os nematoides anisaquídeos estavam presentes em $27,3 \%, 64 \%$ e $92,9 \%$, respectivamente, das amostras examinadas. Entretanto, cabe ressaltar que os $92,9 \%$ das amostras positivas observadas por Ramos (2011) foram associadas ao bacalhau salgado seco. A autora também observou a presença dessas larvas no bacalhau demoIhado ultracongelado, com $100 \%$ das amostras positivas. Resultado esse semelhante ao encontrado no presente estudo, no qual $100 \%$ das amostras estavam parasitadas por larvas de anisaquídeos. Além disso, a distribuição e localização das larvas de Anisakis simplex no bacalhau, observada no presente estudo, apresentou similaridade em relação trabalhos supracitados.

As larvas anisaquídeos são encontradas com freqüência em peixes marinhos da costa brasileira (LUQUE, POULIN, 2004; BICUDO et. al., 2005; CARDOSO et al., 2006; TAVARES, LUQUE, 2006; PEREZ et al. 2014). Entretanto, na maioria dos estudos, são registradas ocorrendo sobre o mesentério, na cavidade celomática e nas vísceras dos peixes. Segundo Lymbery e Cheah (2007) e Saad e Luque (2009), a presença de larvas na musculatura dos peixes é própria de algumas espécies de anisaquídeos, como Anisakis simplex e Pseudoterranova decipiens, de reconhecida importância zoonótica, mas a presença de outras espécies de larvas de anisaquídeos, na musculatura somática, pode ser consequência de migração post-mortem ou durante o processo de congelamento. Em peixes provenientes do litoral do Estado do Rio de Janeiro, essas larvas foram observadas na musculatura do congro rosa (Genypterus brasiliensis) (KNOFF et al., 2004; 2007), do linguado (Paralichthys isosceles) (FELIZARDO et. al., 2009) e do pargo (Pagrus pagrus) (SAAD, LUQUE, 2009).

Os vermes adultos de Anisakis e de Pseudoterranova são encontrados principalmente no estômago e intestino de cetáceos (baleias, golfinhos e botos) e de pinípedes (focas, leões marinhos e morsas) (Pinnipedia). Os ovos produzidos pelos adultos são eliminados com as fezes desses hospedeiros definitivos. Após o processo de desenvolvimento embrionário, os ovos, contendo as larvas $L_{1} e$ $\mathrm{L}_{2}$, ou as larvas livres nadantes, são ingeridos por uma ampla variedade de pequenos crustáceos e de moluscos que atuam como hospedeiros intermediários, em cujo interior se formam as larvas de terceiro estágio $\left(L_{3}\right)$. Os peixes ingerem os crustáceos, contendo $\mathrm{L}_{3}$, e atuam como hospedeiros de transporte ou paratênicos, onde se acumulam e encistam as larvas $L_{3}$ a espera da predação pelos hospedeiros definitivos. Os mesmos podem ser ingeridos por peixes maiores e, nesse caso, ocorre apenas a trans- 
ferência do verme de um hospedeiro para outro sem desenvolvimento ou podem ser ingeridos pelos hospedeiros definitivos completando o amadurecimento, acasalamento e consequente produção de ovos. 0 ser humano é considerado um hospedeiro acidental, no qual a larva ingerida com o pescado cru ou mal cozido não chega à maturidade (ACHA, SZYFRES, 2003; POZIO, 2013).

A parasitose em humanos, causada pelas larvas de família Anisakidae, é denominada de anisaquiose, cujos agentes etiológicos mais comuns são os nematoides dos gêneros Anisakis, Pseudoterranova (= Porrocaecum, Terranova, Phocanema) e Contracaecum (SMITH, WOOTTEN, 1978; ACHA, SZYFRES, 2003). Entretanto, recentes estudos utilizam o termo pseudoterranovose (pseudoterranovosis) para a parasitose causada pelos nematoides do gênero Pseudoterranova (MEASURES, 2014). Historicamente, a anisaquiose em humanos foi registrada em países como Japão, Alasca e Holanda. Entretanto, atualmente tem ocorrido em países das Américas, na Europa e na Ásia (CRUZ et al., 2010; PONTONE et al., 2012; MATTIUCCI et al.; 2013; MEASURES, 2014), reforçando o perfil de ser uma zoonose emergente.

A manifestação clínica da anisaquiose apresenta várias formas. As larvas podem permanecer no lúmen do estômago e do intestino, sem penetrar nos tecidos, e produzir uma infecção que, na maioria das vezes, é assintomática. Nesse caso, a infecção é descoberta após a expulsão das larvas vivas pelo vômito ou pelas fezes. Quando as larvas penetram no tecido, pode chegar à submucosa gástrica ou intestinal, causando edema, úlcera e hemorragia (ACHA, SZYFRES, 2003). Segundo Smith (1999), as manifestações clínicas presentes na anisaquiose podem ser classificadas como: (1) forma clínica não invasiva, quando a larva não penetra na mucosa, normalmente apresentando um quadro assintomático; (2) forma clínica invasiva, com a penetração da larva na região orofaríngea, no trato gastrintestinal e extra-trato digestório, com manifestações moderadas a severas; (3) forma clínica alérgica. Nesse último caso, a manifestação é ocasionada tanto pelos antígenos somáticos, quanto pelos materiais excretados pelas larvas que produzem reações de hipersensibilidade imediata mediada por IgE. Por isso, não é necessário que a larva esteja viva para que se desenvolvam as reações de hipersensibilidade. Indivíduos sensibilizados podem apresentar prurido, urticária, asma e/ou angioedema. Apesar da controvérsia, existe a descrição da possibilidade de urticária crônica. Tem sido descritos casos de anafilaxia em pacientes hipersensibilizados com níveis elevados de IgE. As manifestações alérgicas ocorrem, em geral, duas horas após infecção e podem cursar sozinhas ou associadas com as alterações gastrintestinais (TRUJILLO et al., 2002; ACHA, SZYFRES, 2003; PONTONE et al., 2012; JUNIOR et al., 2013).

A infecção humana pode ser prevenida através da abstenção da ingestão do pescado cru. A maioria das espécies de anisaquídeos de potencial zoonótico, para o homem, morre quando expostas a temperaturas de $-20^{\circ} \mathrm{C}$, por 72 horas ou através da cocção a $70^{\circ} \mathrm{C}$, por pelo menos um minuto. Também é eficaz o processo de conservação em solução concentrada de sal que alcancem todas as partes do pescado. Além disso, preconiza-se a imediata evisceração do pescado depois da captura para evitar que as larvas de Anisakis migrem do intestino para musculatura (SMITH, WOOTTEN, 1975; ACHA, SZYFRES, 2003).

Destarte, os resultados obtidos no presente artigo, no qual foram encontradas larvas de Anisakis simplex em todas as amostras de bacalhau analisadas, indicam que o produto estava impróprio para consumo e que podem representar significativo risco à saúde humana, em virtude das questões mencionadas anteriormente. Nesse caso, recomenda-se que seja realizada a rigorosa análise visual dos peixes, com descarte de espécimes parasitados. $\mathrm{Na}$ indústria, essa análise pode incluir a utilização da mesa de inspeção "candling table" para análise de amostragem do produto filetado. Além disso, conforme sugerido por Junior et al. (2013), a população sensibilizada deve evitar consumir o pescado cru ou pouco processado, evitar o peixe pequeno e inteiro, além daqueles em que se tiverem dificuldades de reconhecimento de sua origem. Sempre avisar ao dono do estabelecimento que é alérgico ao Anisakis e se assegurar que o pescado reúne garantias suficientes de ausência de contaminação pelo parasito. 
Prado e Capuano (2006) sugerem que sejam adotadas ações mais efetivas e exigentes dos órgãos competentes, assim como a adoção de políticas mais severas, com a exigência de lotes já congelados e isentos desses parasitas. Tais diretrizes são de grande relevância para garantir a qualidade higiênicosanitária desses produtos e a segura importação e comercialização do bacalhau.

\section{AGRADECIMENTO}

Agradecemos ao Centro Universitário de Volta Redonda (UniFOA) pelo apoio financeiro.

\section{REFERÊNCIAS}

ACHA, P. N.; SZYFRES, B. Zoonosis y enfermedades transmisibles comunes al hombre y a los animales:

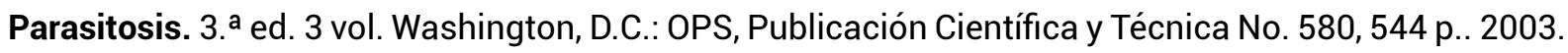

AMATO, J. F. R., BOEGER, W. A.; AMATO, S. B. Protocolos para Laboratório - Coleta e Processamento de Parasitos de Pescado. Imprensa Universitária, Universidade Federal Rural do Rio de Janeiro, 81 p., 1991.

BICUDO, A. J. A.; TAVARES, L. E. R.; LUQUE, J. L. Larvas de Anisakidae (Nematoda: Ascaridoidea) parasitas da cabrinha Prionotus punctatus (Bloch, 1793) (Osteichthyes: Triglidae) do litoral do Estado do Rio de Janeiro, Brasil. Revista Brasileira de Parasitologia Veterinária, v. 14, n. 3, p. 109-118, 2005.

BRASIL. Classificação de risco dos agentes biológicos. Ministério da Saúde, Secretaria de Ciência, Tecnologia e Insumos Estratégicos, Departamento do Complexo Industrial e Inovação em Saúde. - 2. ed. - Brasília: Editora do Ministério da Saúde, 41p., 2010.

BUSH, A.O.; LAFFERTY, K.D.; LOTZ, J.M.; SHOSTAK, A.W. Parasitology meets ecology on its own terms: Margolis et al. revisited. Journal of Parasitology., v. 83, n. 4, p. 575- 583, 1997.

CAPUANO, D. M.; OKINO, M. H. T.; MATTOS, H. R. M.; TORRES, D. M. A. G. V. Diphyllobothriasis: a case report of a human infection in Ribeirão Preto, SP, Brazil, Revista Brasileira de Análises Clínicas, v. 39, p. 163-164, 2007.

CARDOSO, T. P.; SALGADO, R. L.; ANDRADE, P. F.; SÃO CLEMENTE, S. C.; LIMA, F. C. Nematóides da família Anisakidae e cestóides da ordem Trypanorhyncha em peixes teleósteos comercializados no estado do Rio de Janeiro. Revista Brasileira de Ciências Veterinárias, v. 13, n. 2, p. 98-101, 2006.

CHIEFFI P.P., LEITE O.H., DIAS R.M.D.S., TORRES D.M.A.G.V. \& MANGINI A.C.S. Human parasitism by Phagicola sp. (Trematoda: Heterophyidae) in Cananéia, SP, Brazil. Revista do Instituto de Medicina Tropical de São Paulo, v. 32, p. 285-288, 1990.

CHIEFFI, P. P.; GORLA, M. C. O.; TORRES, D. M. A. G. V.; DIAS, R. M. D. S.; MANGINI, A. C. S.; MONTEIRO, A. V.; WOICIECHOVSKI, E. Human infection by Phagicola sp. (Trematoda, Heterophyidae) in the municipality of Registro, São Paulo State, Brazil. Journal of Tropical Medicine and Hygiene., v. 95, p. 346-348, 1992.

CRUZ, A. R.; SOUTO, P. C. S.; FERRARI, C. K. B.; ALLEGRETTI, S. M.; ARRAIS-SILVA, W. W. Endoscopic imaging of the first clinical case of Anisakidosis In Brazil. Scientia Parasitologica, v. 11, n. 2, p. 97-100, 2010. 
EDUARDO, M. B. P.; SAMPAIO, J. L. M.; GONÇALVES, E. M. N.; CASTILHO, V. L. P.; RANDI, A. P.; THIAGO, C.; PIMENTEL, E. P.; PAVANELLO, E. I.; COLLEONE, R. P.; VIGILATO, M. A. N.; MARSIGLIA, D. A. P.; ATUI, M. B.; TORRES, D. M. A. G. V. Diphyllobothrium spp.: um parasita emergente em São Paulo, associado ao consumo de peixe cru - sushis e sashimis. Boletim Epidemiológico Paulista, v. 2, n. 15, p. 1-5, 2005a.

EDUARDO, M. B. P.; SAMPAIO, J. L. M.; SUSUKI, E.; CÉSAR, M. L. V. S.; GONÇALVES, E. M. N.; CASTILHO, V. L. P.; ALBUQUERQUE, S. M. S. R.; PAVANELLO, E. I.; VIGILATO, M. A. N.; LÍRIO, V. S.; MANTESSO, I. S.; ZENEBON, O.; MARSIGLIA, D. A. P.; ATUI, M. B.; RODRIGUES, R. S. M.; RODRIGUES, R. M. M. S.; TORRES, D. M. A. G. V.; LATORRE, W. C., FORTALEZA, C. M. C. B. Investigação epidemiológica do surto de difilobotríase. Boletim Epidemiológico Paulista, v. 2, n. 17, p. 1-20, 2005b.

EIRAS, J. C.; TAKEMOTO, R. M.; PAVANELLI, G. C. Métodos de estudo e técnicas laboratoriais em parasitologia de peixes: 2. ed. Universidade Estadual de Maringá, Maringá, p.171, 2000.

EMMEL, V. E.; INAMINE, E.; SECCHI, C.; BRODT, T. C. Z.; AMARO, M. C. O.; CANTARELLI, V. V.; SPALDING, S. Diphyllobothrium latum: relato de caso no Brasil. Revista da Sociedade Brasileira de Medicina Tropical., v. 39, p. $82-84,2006$.

FELIZARDO, N. N.; KNOFF, M.; PINTO, R. M.; GOMES, D. C. Larval anisakid nematodes of the flounder, Paralichthys isosceles Jordan, 1890 (Pisces: Teleostei) from Brazil. Neotropical Helminthology., v. 3, n. 2, p. $57-64,2009$.

JUNIOR, I F.; VERÍCIMO, M.; SÃO CLEMENTE, S. C.; TEIXEIRA, G. Anisaquiose Humana. Revista de Pediatria SOPERJ, v. 14, n. 1, p. 8-15, 2013.

KNOFF, M.; SÃO CLEMENTE, S. C.; FONSECA, M. C. G.; ANDRADA, C. D. G.; PADOVANI, R. E. S.; GOMES, D. C. Anisakidae parasitos de congro-rosa, Genypterus brasiliensis Regan, 1903 comercializados no estado do Rio de Janeiro, Brasil de interesse na saúde pública. Parasitología Latinoamericana, v. 62, p. $127-133,2007$.

KNOFF, M.; SÃO CLEMENTE, S. C.; GOMES, D. C.; PADOVANI, R. E. S. Primeira ocorrência de larvas de Anisakis sp. na musculatura de congro-rosa, Genypterus brasiliensis Regan, 1903. Revista Brasileira de Ciências Veterinárias., v. 11, n. 1/2, p. 119-120, 2004.

LEITE, O. H. M.; HIGAKI, Y.; SERPENTINI, S. L. P.; CARVALHO, S. A.; NETO A. V.; TORRES, D. M.; DIAS, R. M.; CHIEFFI, P. P. Infecção por Clonorchis sinensis em imigrantes asiáticos no Brasil: tratamento com praziquantel. Revista do Instituto de Medicina Tropical de São Paulo, v. 31, p., 416-422, 1989.

LUQUE, J. L.; POULIN, R. Use of fish as intermediate hosts by helminth parasites: a comparative analysis. Acta Parasitologica., v. 49, n. 4, p. 353-361, 2004.

LYMBERY, A. J.; CHEAH, F. Y. Anisakid nematodes and anisakiasis. In: MURRELL, K. D.; FRIED, B. (Eds.). Food-Borne parasitic zoonoses: fish and Plant-Borne parasites. New York: Springer, p. 185-207, 2007.

MAFRA, C.; MANTOVANI, C.; BORGES, J. N.; BARCELOS, R. M.; SANTOS, C. P. Morphological and molecular diagnosis of Pseudoterranova decipiens (sensu stricto) (Anisakidae) in imported cod sold in Brazil. Brazilian Journal of Veterinary Parasitology., v. 24, n. 2, p. 209-215, 2015.

MATTIUCCI, S.; FAZII, P.; ROSA, A.; PAOLETTI, M.; MEGNA, A. S.; GLIELMO, A.; ANGELIS, M.; COSTA, A.; MEUCCI, C.; CALVARUSO, V.; SORRENTINI, I.; PALMA, G.; BRUSCHI, F.; NASCETTI, G. Anisakiasis and 
Gastroallergic Reactions Associated with Anisakis pegreffii Infection, Italy. Emerging Infectious Diseases. v. 19, n. 3, p. $496-499,2013$.

MATTOS, D. P. B. G.; LOPES, L. M. S.; VERÍCIMO, M. A.; ALVARES, T. S.; SÃO CLEMENTE, S. C. Anisakidae infection in five commercially important fish species from the State of Rio de Janeiro, Brazil. Revista Brasileira de Medicina Veterinária, v. 36, n. 4, p. 375-379, 2014.

MEASURES, L. N. Anisakiosis and pseudoterranovosis: Reston, Va., U.S. Geological Survey Circular 1393, 34 p., 2014.

OKUMURA, M. P. M.; PEREZ, A. C. A.; SPINDOLA, A. F. Principais zoonoses parasitárias transmitidas por pescado. Revisão. Revista de Educação Continuada, v. 2, n. 2, p. 66-80, 1999.

PEREIRA, A.D.; ATUI, M.B.; TORRES, D.M.A.G.V.; MANGINI, A.C. \& ZAMBONI, C.Q. - Incidência de parasitos da família Anisakidae em bacalhau (Gadus morhua) comercializado no Estado de São Paulo. Revista do Instituto Adolfo Lutz, v. 59, p. 45-49, 2000.

PÉREZ, A. C. A.; MACHADO, T. M.; NEIVA, C. R. P.; FURLAN, E. F.; NETO, M. J. L.; TOMITA, R. Y.; RODRIGUES, M. V.; SILVEIRA, N. F. A.; MORGANO, M. A.; MANTOVANI, D. M. B. Evaluation of seafood quality market in baixada Santista/SP. Boletim do Instituto de Pesca, v. 40, n. 2, p. 167 - 177, 2014.

PETTER AJ, MAILLARD C. Larves d'ascarides parasites de poissons en Méditerranée occidentale. Bulletin du Muséum national d'histoire naturelle, v. 10, p. 347-369, 1988.

PONTONE, S.; LEONETTI, G.; GUAITOLI, E.; MOCINI, R.; MANFREDELLI, S.; CATANIA, A.; PONTONE, P.; SORRENTI, S. Should the host reaction to anisakiasis influence the treatment? Different clinical presentations in two cases. Revista Española de Enfermedades Digestivas. v. 104. n. 11, p. 607-610, 2012.

POZIO, E. Integrating animal health surveillance and food safety: the example of Anisakis. Scientific and Technical Review of the Office International des Epizooties, v. 32, v. 2, p. 487-496, 2013.

PRADO, S. P. T.; CAPUANO, D. M. Relato de nematóides da família Anisakidae em bacalhau comercializado em Ribeirão Preto, SP. Revista da Sociedade Brasileira de Medicina Tropical, v. 39, n. 6, p. :580-581, 2006.

RAMOS, P. Anisakis spp. em bacalhau, sushi e sashimi: risco de infecção parasitária e Alergia. Revista Portuguesa de Ciências Veterinárias, v. 106, p. 87-97, 2011.

ROSSI, G. A. M.; HOPPE, E. G. L.; MARTINS, A. M. C. V.; PRATA, L. F. Zoonoses parasitárias veiculadas por alimentos de origem animal: revisão sobre a situação no Brasil. Arquivos do Instituto Biológico, v.81, n. 3, p. 290-298, 2014.

SAAD, C. D. R.; LUQUE, J. L. Larvas de Anisakidae na musculatura do pargo, Pagrus pagrus, no Estado do Rio de Janeiro, Brasil. Revista Brasileira de Parasitologia Veterinária., v. 18, supl. 1, p. 71-73, 2009.

SAAD, C. D. R.; VIEIRA, F. M.; LUQUE, J. L. Larvas de Anisakidae Skrjabin \& Karokhin, 1945 (Nematoda, Ascaridoidea) em Lophius gastrophysus Miranda-Ribeiro, 1915 (Actinopterygii, Lophiidae) no litoral do Estado do Rio de Janeiro, Brasil. Neotropical Helminthology, v. 6, n. 2, p. 159 - 177, 2012.

SANTOS F.L.N. \& FARO L.B. The first confirmed case of Diphyllobothrium latum in Brazil. Memórias do Instituto Oswaldo Cruz, v. 100, p. 685-686, 2005. 
SANTOS, C. A. M. L. Doenças transmitidas por pescado no Brasil. Revista Brasileira de Medicina Veterinária, v. 32, n. 4, p. 234-241, 2010.

SMITH, J. W. Ascaridoid nematodes and pathology of the alimentary tract and its associated organs in vertebrates, including man: a literature review: Helminthological Abstracts, v. 68, p. 49-96, 1999.

SMITH, J. W.; WOOTTEN, R. Anisakis and anisakiasis: Advances in Parasitology, v. 16, p. 93-163, 1978.

SMITH, J. W.; WOOTTEN, R. Experimental studies on the migration of Anisakis sp. larvae (Nematoda: Ascaridida) into the flesh of herring, Clupea harengus L.. International Journal for Parasitology, v. 5, p. 133-136, 1975.

TAVARES L. E. R.; LUQUE, J. L. Sistemática, biologia e importância em saúde coletiva de larvas de Anisakidae (Nematoda: Ascaridoidea) parasitas de peixes ósseos marinhos do Estado do Rio de Janeiro, Brasil. In: A. T. SILVA-SOUZA (Ed.), Sanidade de Organismos Aquáticos no Brasil. Maringá: Abrapoa, p. 297-328, 2006.

TAVARES, L. E. R.; LUQUE, J. L.; BOMFIM, T. C. B. Human Diphyllobothriasis: reports from Rio de Janeiro, Brazil. Revista Brasileira de Parasitologia Veterinária., v. 14, n. 2, p. 85-87, 2005.

TRUJILLO, M. L.; RODRÍGUEZ, A.; BARA, M. T. G.; MATHEU, V.; HERRERO, T.; RUBIO, M.; ZUBELDIA, J. M.; BAEZA, M. L. Dietary recommendations for patients allergic to Anisakis simplex. Allergologia et immunopathologia, v. 30, n. 6, p. 311-4, 2002. 